progression of $\mathrm{AAV}$, guideline recommendations on diagnosing and managing AAV with guideline-directed medical therapies (GDMTs), strategies for the management of relapsing and refractory disease in AAV and positioning of emerging therapies in the treatment paradigm.

REFERENCES:

[1] www.medscape.org/viewarticle/920320.

[2] Hutton HL, et al. Semin Nephrol 2017;37(5):418-35.

[3] Al-Hussain T, et al. Adv Anat Pathol 2017;24(4):226-34.

Disclosure of Interests: Sarah Mendly Grant/research support from: Supported by an independent educational grant from Vifor Pharma, George Boutsalis Grant/research support from: Supported by an independent educational grant from Vifor Pharma

DOI: 10.1136/annrheumdis-2021-eular.3684

\section{AB0883-HPR CHRONIC RHEUMATIC DISEASE MANAGEMENT AND THE SELF-EFFICACY OF RHEUMATOID ARTHRITIS}

Y. H. Xu ${ }^{1}$, X. Zhang ${ }^{1}$, C. Chen ${ }^{1}$, Y. W. Zou ${ }^{1}$, K. M. Yang ${ }^{1}$, L. Dai ${ }^{1}{ }^{1}$ Sun Yat-Sen Memorial Hospital, Sun Yat-Sen University, Department of Rheumatology, Guangzhou, China

Background: Rheumatoid arthritis (RA) is a common chronic disabling autoimmune disease, which cannot be cured completely at present, and patients need long-term medication treatment. The overall management of RA disease and patient self-management is important. Therefore, we built up a chronic rheumatic disease management team, formed by rheumatoid clinicians, clinical nurses and case managers, to provide "integrity continuity" disease management for RA by hospital on-site and WeChat APP. To this end, we used the arthritis self-efficacy scale-8 (ASES-8) to investigate the effect of the chronic disease management with RA.

Objectives: To explore the effect of chronic rheumatic disease management and the related factors of self-efficacy of RA patients.

Methods: Consecutive outpatients with RA who had been implemented chronic disease management were recruited from January 2019 to January 2020. A questionnaire survey of ASES-8 and clinical data were collected.

Results: 1. Among the 376 RA patients who have completed the survey, mean follow-up time of chronic disease management was $29(8,41)$ months, $35.4 \%$ patients in CDAI remission stage of disease activity, median ASES-8 score was $59(47,70)$, and $61.2 \%$ patients had medium or above medication adherence.

2. Patients with low ASES-8 had higher RA disease activity (including TJC28, SJC28, Pain VAS, PtGA, PrGA, ESR, CRP), more patients with dysfunction and lower medication adherence than those with high ASES-8 (all $P<0.05)$.

3. Patients were divided into CDAI active RA group and in remission group, and those with low ASES-8 in remission group participated in chronic disease management for fewer months than those with high ASES-8 (median 20 vs. $32, P=0.033)$, and the HAQ-DI score was higher $(P<0.05)$. Active RA patients with low ASES-8 had higher disease activity, higher HAQ-DI (median 0.25 vs.0), fewer times of participating in patients' exchange meeting (median 0 vs. 1 ), lower medication adherence (10.8\% vs. $25.9 \%$ ), and more anxiety or depression (57.6\% vs. $31.8 \%$ ) than those with high ASES-8 (all $P<0.05$, Table 1 below).
Conclusion: The rheumatic chronic disease management team can help RA patients achieve a higher remission rate by continuous chronic disease management through hospital on-site and the Internet, and can help patients with good self-efficacy and medication adherence. In addition to disease control, we should pay attention to functional limitations and problems of back to work with RA patients in remission, while should pay attention to activity participation, medication adherence and emotional problems with active RA in further practice.

Disclosure of Interests: None declared

DOI: 10.1136/annrheumdis-2021-eular.3785

\section{HPR Patients' perspectives, functioning and health (descriptive: qualitative or quantitative)}

\section{AB0884-HPR PERSONALISED TREATMENT GOALS IN PATIENTS WITH IMMUNE MEDIATED INFLAMMATORY DISEASE}

S. Fadaei ${ }^{1}$, H. Van Os-Medendorp ${ }^{1}$, A. Sloeserwij ${ }^{1}$, V. Sigurdsson ${ }^{2}$, J. M. Van Laar ${ }^{1}$, J. Spierings ${ }^{1} .{ }^{1}$ UMC Utrecht, Rheumatology \& Clinical Immunology, Utrecht, Netherlands; ${ }^{2}$ UMC Utrecht, Internal Medicine \& Dermatology, Utrecht, Netherlands

Background: Immune mediated inflammatory diseases (IMIDs) often have a chronic character and large impact on daily life of patients. In order to provide patient-centred care, insight in personal goals is a key issue.

Objectives: The aim of this study was to evaluate treatment goals of patients with IMIDs over time.

Methods: Patients with IMIDs were invited to complete a self-designed questionnaire about lifestyle, personal treatment goals and perceived care at their first visit to our centre and one year after referral. Clinical information was collected through the electronic patient record system.

Results: 41 (30\%) patients of the 137 invited, completed both questionnaires. Participants had a mean age of 45.6 years (SD 13.4), $\mathrm{N}=35(83 \%)$ was female, mean disease duration was 12.7 years (SD 12.7). Patients were diagnosed with connective tissue disease (CTD, $n=19 ; 45 \%$ ), antiphospholipid syndrome (APS, $n=10 ; 24 \%)$, inflammatory arthritis $(n=7 ; 17 \%)$ and psoriasis $(n=7 ; 17 \%)$. We identified six categories of treatment goals: disease related, daily functioning, lifestyle, emotional wellbeing. Figure 1 shows the reported treatment goals at baseline and follow-up. Most frequently mentioned were goals related to disease, lifestyle and daily functioning. At follow-up we observed a vast increase in reported goals related to psychological wellbeing (from $5 \%$ to $16 \%$ ). At baseline patients with APS chose lifestyle-related goals more often than other patients $(R R=3.2 ; p=0.015)$. At follow-up patients with CTDs reported disease related-goals less often than other patients $(R R=0.4$ $\mathrm{p}=0.002$ ). Patients did not report significant progress in reaching their goals on a Likert scale of $1-10$. However, in $90 \%$ at first visit and in $83 \%$ of patients at follow-up, patients reported that the provided care was addressing issues they personally prioritized.

Conclusion: IMID patients' most frequent treatment goals relate to disease activity, lifestyle and daily functioning. Patients frequently change their treatment goals during the first year of treatment. Better understanding of

Table 1. Comparison of ASES in different RA disease activity

\begin{tabular}{|c|c|c|c|c|c|c|}
\hline \multirow[t]{2}{*}{ Characteristics } & \multicolumn{3}{|l|}{ CDAI remission } & \multicolumn{3}{|l|}{ CDAl active } \\
\hline & $\begin{array}{l}\text { Low ASES (ASES } \leq 59 \text {, } \\
n=36)\end{array}$ & $\begin{array}{l}\text { High ASES (ASES }>59 \text {, } \\
\mathrm{n}=97 \text { ) }\end{array}$ & $P$ & $\begin{array}{l}\text { Low ASES (ASES } \leq 59 \text {, } \\
n=158)\end{array}$ & $\begin{array}{l}\text { High ASES (ASES }>59 \text {, } \\
n=85 \text { ) }\end{array}$ & $P$ \\
\hline Female, n (\%) & $32(88.9)$ & $81(83.5)$ & 0.440 & $137(86.7)$ & $74(87.1)$ & 0.939 \\
\hline Age, yrs, $\pm s$ & $47.4 \pm 11.7$ & $46.0 \pm 12.5$ & 0.574 & $49.8 \pm 12.9$ & $49.7 \pm 12.7$ & 0.933 \\
\hline Disease duration, month, median (IQR) & $48(24,94)$ & $60(35,96)$ & 0.560 & $75(36,120)$ & $72(36,120)$ & 0.625 \\
\hline \multicolumn{2}{|c|}{$\begin{array}{l}\text { Duration of chronic disease management, month,20 }(6,39) \\
\text { median (IQR) }\end{array}$} & $32(13,41)$ & 0.033 & $29(6,41)$ & $29(5,42)$ & 0.766 \\
\hline \multicolumn{2}{|c|}{$\begin{array}{l}\text { Attend patients' exchange meeting, times, median6 }(4,10) \\
\text { (IQR) }\end{array}$} & $8(6,13)$ & 0.673 & $0(0,2)$ & $1(0,4)$ & 0.035 \\
\hline Incapable to work, n (\%) & $11(30.6)$ & $15(15.5)$ & 0.051 & $46(29.1)$ & $18(21.2)$ & 0.180 \\
\hline \multicolumn{2}{|l|}{ Morisky medication adherence scale } & & 0.857 & & & 0.002 \\
\hline Low adherence, $n(\%)$ & $12(33.3)$ & $30(30.9)$ & & $78(49.4)$ & $26(30.6)$ & \\
\hline Medium adherence, $\mathrm{n}(\%)$ & $15(41.7)$ & 38 (39.2) & & $63(39.9)$ & 37 (43.5) & \\
\hline High adherence, $\mathrm{n}(\%)$ & $9(25.0)$ & 29 (29.9) & & $17(10.8)$ & $22(25.9)$ & \\
\hline 28TJC, median (IQR) & $0(0,0)$ & $0(0,0)$ & 0.148 & $3(1,5)$ & $1(1,3)$ & 0.003 \\
\hline 28SJC, median (IQR) & $0(0,0)$ & $0(0,0)$ & 0.734 & $1(0,3)$ & $1(0,2)$ & 0.044 \\
\hline PtGA, median (IQR) & $0(0,0)$ & $0(0,0)$ & 0.302 & $4(2,5)$ & $2(1,3)$ & $<0.001$ \\
\hline PrGA, median (IQR) & $0(0,0)$ & $0(0,0)$ & 0.302 & $4(2,5)$ & $2(1,3)$ & $<0.001$ \\
\hline Pain VAS, median (IQR) & $0(0,0)$ & $0(0,1)$ & 0.800 & $3(2,4)$ & $2(1,2)$ & $<0.001$ \\
\hline CRP, (mg/L), median (IQR) & $3(3,3)$ & $3(3,3)$ & 0.833 & $3(3,10)$ & $3(3,6)$ & 0.422 \\
\hline HAQ-DI, median $\left(P_{5}, P_{95}\right)$ & $0(0,0.1625)$ & $0(0,0.0125)$ & 0.016 & $0.25(0,1.25)$ & $0(0,0.837)$ & $<0.001$ \\
\hline Anxiety or depression, ${ }^{5}(\%)$ & $12(33.3)$ & $24(24.7)$ & 0.322 & $91(57.6)$ & $27(31.8)$ & $<0.001$ \\
\hline
\end{tabular}

